\title{
Tracheostomy in pediatric critically ill patients
}

\author{
Gustavo Caprotta*, Martin Tiszler, Ximena Chávez and Judith Frydman \\ Pediatric Intensive Care Unit, Hospital for Trauma and Emergency Dr. Federico Abete, Malvinas \\ Argentinas Buenos Aires, Argentina
}

Received 23 April 2012

Revised 17 June 2012

Accepted 6 August 2012

\begin{abstract}
The indications for and timing of tracheostomy in children have changed significantly over recent years, and no consensus has been gathered in that regard. The purpose of this study is to present a series of critically ill patients who required a tracheostomy. All critically ill patients required a tracheostomy between 1 June 2009 and 31 March 2012. It is a retrospective, observational, descriptive study. A total of 18 patients underwent tracheostomy during the period under study. The most common indication was neuromuscular compromise. The average duration of mechanical ventilation before placement of a tracheostomy was 23.8 days (0-58 days). The complications observed were minor, and no patients died from tracheostomy-related causes. All parents were trained in airway management and cardiopulmonary resuscitation. There were no serious tracheostomy-related complications in critically ill pediatric patients. The procedure was effective in the management of patients with respiratory failure, patients with neuromuscular compromise and children with upper airway obstruction.
\end{abstract}

Keywords: Tracheostomy, intensive care unit, pediatrics

\section{Introduction}

In the past 20 years (yr), advances in medicine and technology have led to significant improvements in survival among children and newborns with previously fatal conditions. Many times, survivors are discharged from the intensive care unit with some degree of technology dependence (chronic mechanical ventilation, gastrostomies, tracheostomies, etc.). One of the factors that contribute to the patients' discharge home or to less restrictive levels of care is the performance of a tracheostomy. This procedure provides an interface for prolonged mechanical ventilation (MV) or is used when long-term oxygen therapy is required in patients with upper airway injuries, after-effects of viral infections on the lungs and/or neuromuscular disease. The indications for and timing of tracheostomy have changed over time

*Corresponding author: Gustavo Caprotta, Unidad de Cuidados Intensivos Pediátricos, del Hospital de Trauma y Emergencia Dr. Federico Abete, Municipio de Malvinas Argentinas, Provincia de Buenos Aires. Nogoya 4084 PB 2 Argentina. Tel.: +54911 155061 3064; Fax: 541144699600 int. 422; E-mail: gcapro@gmail.com.
$[1,2]$. This has promoted better tolerance of MV and earlier weaning, lowering the need for sedation and ana lgesia, reducing intercurrent infections associated with MV and contributing to nutritional recovery $[2,3]$. There is some controversy concerning the indications for and timing of tracheostomy in pediatric patients, particularly in those patients requiring prolonged MV as a result of an acute respiratory disease [4]. The decision for tracheostomy is, however, more clear in the case of patients with neuromuscular disease and patients with upper airway injuries. The purpose of this work is to describe a case series involving critical patients who required tracheostomy, specifying indications, surgical timing and complications, and to compare it against other publications.

\section{Materials and methods}

This is a descriptive, observational, and retrospective study. All the patients requiring a new tracheostomy between June 1, 2009 and March 31, 2012 who were admitted to the pediatric intensive care unit (PICU) of 
hospital de trauma y emergencia Dr. Federico Abete (Dr. Federico Abete trauma and emergency hospital), located within the Malvinas Argentinas Municipality, in the Province of Buenos Aires, were enrolled. This is a polyvalent (general) PICU that has 20 beds and admits patients up to $14 \mathrm{yr}$ of age.

\section{Results}

During the period under discussion, 1330 patients were admitted to our unit, 402 of whom required MV. A tracheostomy was performed in $4.4 \%$ of ventilated patients $(\mathrm{n}=18)$ (Table 1$)$. Patients were classified into three different subgroups based on the indication for tracheostomy: neuromuscular causes $44.4 \%(n=8)$, upper airway obstruction $33.3 \%(n=6)$, and the remaining $22.2 \%(n=4)$ after a period of prolonged MV due to pulmonary disease. (Fig. 1) Distribution by gender was $67 \%$ male and $33 \%$ female, with ages ranging from 7 days of life to $13 \mathrm{yr}$ of age (average age 48 months). Fifty-five $(55 \%)$ percent of patients were younger than 1 year. An emergency tracheostomy was performed at the unit. The other 17 procedures were performed at the operating room by the pediatric surgery service at our hospital. Previous endoscopy was performed in 10 out of the 18 tracheostomized patients (55\%), showing pathologic results in $90 \%$ of the cases. All endoscopies were indicated after at least three extubation failures with signs of severe respiratory difficulty after a successful spontaneous breathing trial [5-7]. Injuries noted on endoscopy included: defect in the back wall of the larynx, significant laryngeal edema, posterior fusion of the vocal folds, subglottic stenosis, accessory tracheal bronchus, and bilateral paralysis of the vocal folds (Table 1). Patients whose indication for tracheostomy was neuromuscular disease $(45 \%, \mathrm{n}=8)$ did not undergo endoscopy. None of these patients had proper airway protection. Among them were three patients with nonprogressive chronic encephalopathy, one with longitudinal myelitis, one with severe cranioencephalic trauma, one with infant botulism, one with adrenoleukodystrophy, and one with merosin-negative muscular dystrophy. The average duration of MV prior to tracheostomy was 23.8 days ( $0-58$ days), including days of non-invasive ventilation, whereas in the subgroup of patients with prolonged MV for pulmonary disease, the average duration was 49.5 days (44-58 days), including days of noninvasive ventilation. There were no complications during the immediate postoperative period. In total, six adverse events were reported. A total of $16.6 \%(n=3)$ of patients experienced an episode of accidental decannulation, the most common complication in our series. A total of $11.1 \%(n=2)$ experienced an episode of tube obstruction, and one patient $(5.5 \%$ ) experienced granuloma at stoma as a remote complication. There were no fatal complications. Two patients died in this series, but neither death was related to the tracheostomy. Both patients had severe neurological damage, which was associated with complex congenital heart defect in one of the cases (double outlet left ventricle hypoplasia with univentricular physiology, palliative surgery, and hypoxic-ischemic encephalopathy). In all (100\%) cases, parents were trained in comprehensive care of the tracheostomized patient by the PICU staff, primarily including respiratory therapists, nurses and doctors. This training included suctioning techniques, care of the stoma, and changing the device at least three times by each parent. Prior to discharge from the unit, all parents completed a course on pediatric cardiopulmonary resuscitation focused on the special needs of children with tracheostomy.

\section{Discussion}

Severe injuries to the tracheal mucous membrane have been reported from day 7 to day 10 of endotracheal intubation $[2,8]$. This has led to early consideration of tracheostomy in adult patients. In 2005, Durbin [2] stated that tracheostomy should be considered in every patient who will require prolonged $\mathrm{MV}$; this decision should be made within 7-10 days of mechanical breathing support. Recent publications propose a protocol to undergo tracheostomy within 5-7 days after MV [9]. Given the anatomical differences in children's airways, greater periods of time are considered in pediatrics. In 1992, Puhakka et al. [10] specified a period of 64 days of MV before the tracheostomy, other publications state 37.5 days [11], and Kremer et al. [1] noted an average of 31 days (2-134 days). Our series is closer to this last number, with an average of 23.8 days. In the small number of protocols published, endoscopy does not play an important role when deciding on the procedure. The indication is made by considering elements among which endoscopy is not always included. The prevailing criteria are the individual assessment of each patient and the medical criteria of the treating group [12]. Major influential factors are the cause for initiating MV (laryngeal trauma, epiglottitis, neuromuscular disease, need for prolonged MV, etc.) and the prognosis of the condition in question. Congenital upper airway anomalies resulting in a difficult airway that cannot be 
G. Caprotta et al. / Tracheostomy in critically ill patients

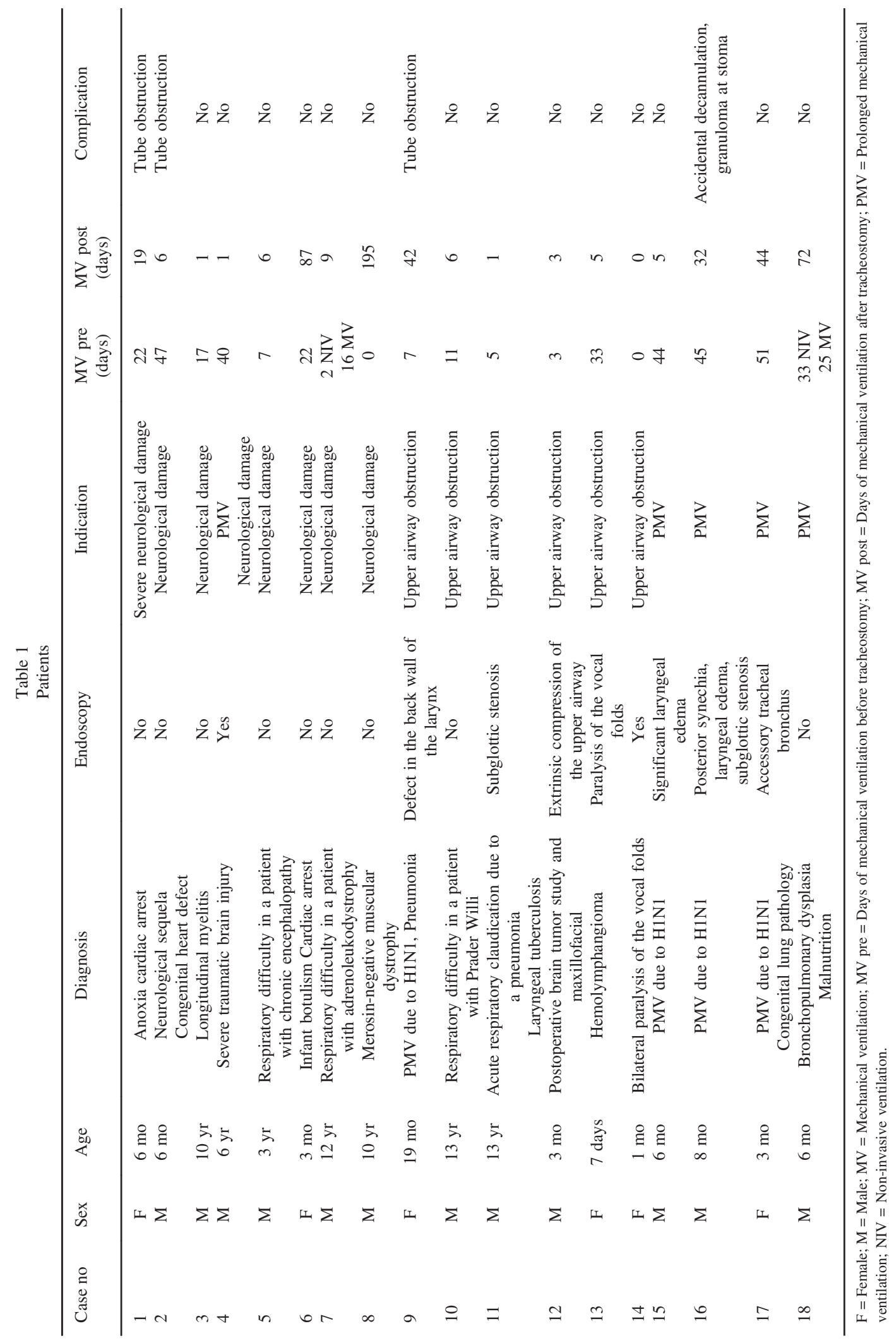

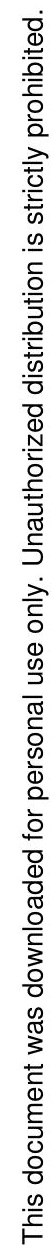




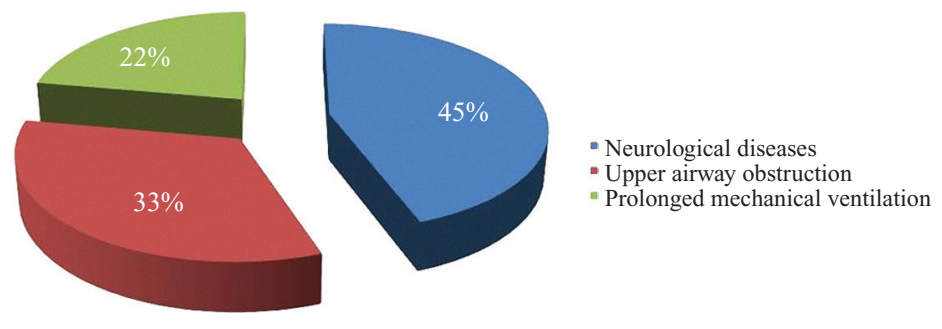

Fig. 1. Indications for tracheostomy.

secured by other means frequently necessitate transient or permanent tracheostomy, especially in infants and young children with compromised nasal breathing [13]. Chronic lung disease of infancy and complex congenital heart disease are other important entities associated with the occasional need for tracheostomy [13]. As pointed out by Litman et al., autopsy studies in children with prolonged intubation demonstrate damage to the entire larynx, with the most severe damage often occurring at the level of the vocal cords [14]. The tracheostomy is being instituted for long-term respiratory support and/or pulmonary toilet. They are often temporary measures until the infant has outgrown his or her susceptibility to critical airway compromise. These patients are intubated for prolonged periods of time in intensive care units, often without muscle relaxants, and whose vocal cords could be vigorously moving and pushing against the endotracheal tube during respiratory cycle and especially during coughing. In our series, we performed endoscopies in all patients who experienced multiple extubation failures despite undergoing a successful spontaneous breathing trial. These endoscopies were extremely helpful, as they provided an anatomical element that explained the cause of previous failures, thus tipping the balance in favor of the indication for tracheostomy. In these patients, tracheostomy would allow recovery of the injured airway by breaking the irritation cycle caused by the endotracheal tube, and contribute to the total recovery of the patient. In those neuromuscular patients who had no prospect of weaning from MV due to their underlying condition, endoscopy was not performed because, regardless of the findings of the endoscopy, the decision to perform a tracheostomy was based on non-anatomical aspects of the airway. There were no complications during the immediate postoperative period or before the first scheduled tube exchange, which was routinely performed at 7 days postoperative. The incidence of complications did not result in any deaths.
The complications observed were minor and resolved rapidly. Consistent with the literature published to date, accidental decannulation was the most common complication in our series [1, 15-17]. The remote complication (granuloma at stoma) showed great progress with local medical treatment (topical silver nitrate). There was no difficulty in properly ventilating tracheostomized patients. In some cases (particularly, upper airway obstruction), the tracheostomy allowed successful weaning from MV within a few days, whereas in other cases weaning from positive pressure ventilation required more gradual strategies [18]. Neuromuscular patients were weaned only upon improvement of the underlying condition (longitudinal myelitis, infant botulism). Parents of patients were all helpful and motivated to participate in the care of their tracheostomized children. Thanks to the support of the psychology, social services and kinesiology areas, they gradually accepted their children's new condition and took on more and more new tasks. In addition to this, cardiopulmonary resuscitation training gave them a sense of confidence to face the new family situation they would find themselves in after discharge from the PICU [19, 20]. After a period of inpatient stay at the pediatric ward, patients were discharged home on an outpatient monitoring program targeting areas such as pediatrics, surgery, endoscopy, pulmonology, social services, psychology, neurology (as appropriate) for follow-up and scheduling timely decannulation. Few children are now tracheotomized for airway emergencies; instead, the procedure is mostly performed for long-term problems. As a consequence, there is in most clinical situations no unity of approach, and the indication for tracheostomy is influenced by multiple circumstantial factors [13]. It is well known that there are potential risk factors for postintubation damages, and namely the underlying disease requiring endotrachel intubation, the age and body weight at that moment, the duration and number of endotracheal intubations, the absence of sedation and the occurrence 
of infectious, hypotensive or hypoxic events during this period as well as traumatic intubations [21]. Some authors propose that the maximum time that a patient may be intubated depends on weight and age. They propose a maximum of 8 weeks in infants and two in children. However, the valuation of this period depends on the real possibilities of extubation of each child and the evolution during the period he has remained intubated. The risk of laryngotracheal complications is always present and increases over time and when added factors such as hypoxia or low output appear [22]. There is no consensuses on which are the proper indications for and timing of tracheostomy in pediatric critical patients. In our series, tracheostomies had no serious complications and allowed better handling of respiratory patients, patients with neuromuscular disease and children with upper airway obstruction. We consider it important to have evidencebased guidelines available in the future to assist intensivists in making such an important decision.

\section{References}

[1] Kremer B, Botos-Kremer AI, Eckel HE, Schlöndorff G. Indications, complications, and surgical techniques for pediatric tracheostomies-an update. J Pediatr Surg 2002;37(11):1556-62.

[2] Durbin CG Jr. Indications for and timing of tracheostomy. Respir Care 2005;50(4):483-7.

[3] Salcedo O, Frutos-Vivar F. Tracheostomy in ventilated patients. What do we do it for?. Med Intensiva 2008;32(2):91-3 (in Spanish).

[4] Yoon P. The infant tracheostomy. Oper Tech Otolaryngol 2005;16(3):183-6.

[5] Mallick A, Bodenham AR. Tracheostomy in critically ill patients. Eur J Anaesthesiol 2010;27(8):676-82.

[6] Farias JA, Alía I, Esteban A, Golubicki AN, Olazarri FA. Weaning from mechanical ventilation in pediatric intensive care patients. Intensive Care Med 1998;24(10):1070-5.

[7] Dursun O, Ozel D. Early and long-term outcome after tracheostomy in children. Pediatr Int 2011;53(2):202-6.
[8] Groves DS, Durbin CG Jr. Tracheostomy in the critically ill: indications, timing and techniques. Curr Opin Crit Care 2007;13(1):90-7.

[9] Freeman BD, Kennedy C, Robertson TE, Coopersmith CM, Schallom M, Sona C, et al. Tracheostomy protocol: experience with development and potential utility. Crit Care Med 2008; 36(6):1742-8.

[10] Puhakka HJ, Kero P, Valli P, Iisalo E. Tracheostomy in pediatric patients. Acta Paediatr 1992;81(3):231-4.

[11] Graf JM, Montagnino BA, Hueckel R, McPherson ML. Pediatric tracheostomies: a recent experience from one academic center. Pediatr Crit Care Med 2008;9(1):96-100.

[12] Zgoda M, Berger R. Tracheostomy in the critically ill patient: Who, when, and how?: A review. Clin Pulm Med 2006;13(2): 111-20.

[13] Trachsel D, Hammer J. Indications for tracheostomy in children. Paediatr Respir Rev 2006;7(3):162-8.

[14] Litman RS, Weissend EE, Shibata D, Westesson Pl. Developmental changes of laryngeal dimensions in unparalyzed, sedated children. Anesthesiology 2003;98(1):41-5.

[15] Fraga JC, Souza JC, Kruel J. Pediatric tracheostomy. J Pediatr (Rio J) 2009;85(2):97-103 (in English, Portuguese).

[16] Carr MM, Poje CP, Kingston L, Kielma D, Heard C. Complications in pediatric tracheostomies. Laryngoscope 2001;111 (11 Pt 1):1925-8.

[17] Itamoto CH, Lima BT, Sato J, Fujita RR. Indications and complications of tracheostomy in children. Braz J Otorhinolaryngol 2010;76(3):326-31.

[18] Caprotta G, Crotti PG, Frydman J. Tracheostomy and mechanical ventilation weaning in children affected by respiratory virus according to a weaning protocol in a pediatric intensive care unit in Argentina: an observational restrospective trial. Ital J Pediatr 2011;37:5.

[19] Botto H, Nieto M, Zanetta A, Rodríguez H. Home care of tracheotomyzed child. Arch Argent Pediatr 2008;106(4):351-6 (in Spanish).

[20] Engels PT, Bagshaw SM, Meier M, Brindley PG. Tracheostomy: from insertion to decannulation. Can J Surg 2009;52(5): 427-33.

[21] Meneghini L, Zadra N, Metrangolo S, Narne S, Giusti F. Post-intubation subglottal stenosis in children: risk factors and prevention in pediatric intensive care. Minerva Anestesiol 2000;66(6):467-1 (in Italian).

[22] Pardo Romero G, Pando Pinto JM, Mogollón Cano-Cortés T, Trinidad Ruiz G, Marcos García M, González Palomino A, et al. Paediatric tracheostomy. Acta Otorrinolaringol Esp 2005;56(7): 317-21 (in Spanish). 